\title{
Impact of Using Linear Optimization Models in Dose Planning for HDR Brachytherapy
}

\author{
Åsa Holm, Torbjörn Larsson and Åsa Carlsson Tedgren
}

\section{Linköping University Post Print}

N.B.: When citing this work, cite the original article.

Original Publication:

Åsa Holm, Torbjörn Larsson and Åsa Carlsson Tedgren, Impact of Using Linear Optimization Models in Dose Planning for HDR Brachytherapy, 2012, Medical physics (Lancaster), (39), 2, 1021-1028.

http://dx.doi.org/10.1118/1.3676179

Copyright: American Association of Physicists in Medicine http://www.aapm.org/main.asp

Postprint available at: Linköping University Electronic Press http://urn.kb.se/resolve?urn=urn:nbn:se:liu:diva-67786 


\title{
Impact of using linear optimization models in dose planning for HDR brachytherapy
}

\author{
Åsa Holm*, Torbjörn Larsson*, and Åsa Carlsson Tedgren ${ }^{\dagger \ddagger}$ \\ * Department of Mathematics, \\ Linköping University, \\ SE-581 83 Linköping, Sweden. \\ E-mail: (asa.holm, torbjorn.larsson)@liu.se. \\ ${ }^{\dagger}$ Department of Medical and Health Sciences, \\ Linköping University, \\ SE-581 85 Linköping, Sweden \\ E-mail: asa.carlsson.tedgren@liu.se. \\ ${ }^{\ddagger}$ Swedish Radiation Safety Authority, \\ SE-171 16 Stockholm, Sweden.
}

\begin{abstract}
Purpose: Dose plans generated with optimization models hitherto used in HDR brachytherapy have shown a tendency to yield longer dwell times than manually optimized plans. Concern has been raised for the corresponding undesired hot spots, and various methods to mitigate these have been developed. The hypotheses upon this work is based are a) that one cause for the long dwell times is the use of objective functions comprising simple linear penalties and b) that alternative penalties, as these are piecewise linear, would lead to reduced length of individual dwell times.

Methods: The characteristics of the linear penalties and the piecewise linear penalties are analysed mathematically. Experimental comparisons between the two types of penalties are carried out retrospectively for a set of prostate cancer patients.

Results: When the two types of penalties are compared, significant changes can be seen in the dwell times, while most dose-volume parameters do not differ significantly. On average, total dwell times were reduced by $4.2 \%$, with a reduction of maximum dwell times by $25 \%$, when the alternative penalties were used.

Conclusion: The use of linear penalties in optimization models for HDR brachytherapy is one cause for the undesired long dwell times that arise in mathematically optimized plans. By introducing alternative penalties, a significant reduction in dwell times can be achieved for HDR brachytherapy dose plans. Although various measures for mitigating the long dwell times are already available, the observation that linear penalties contribute to their appearance is of fundamental interest.
\end{abstract}

Keywords: Brachytherapy, Optimization, Treatment planning, Linear programming, Piecewise linear functions. 


\section{Introduction}

High-dose-rate (HDR) brachytherapy is a type of radiotherapy in which a radioactive source is moved through catheters implanted into the volume of the tumour, or close to it. Planning the dose to be delivered involves several steps, one of which is to determine the source dwell time distribution, that is, where and for how long the radioactive source should be stopped. Modern optimization techniques for anatomy-based inverse treatment planning of HDR brachytherapy make it possible to calculate efficiently and automatically source dwell time distributions within implanted catheters, using techniques such as IPSA $^{1}$ or HIPO ${ }^{2}$. The outcome of inverse planning methods for brachytherapy compares favourably or slightly better than manual methods (often referred to as graphical optimization) when evaluated against dosimetric parameters, such as $V_{100}$ for the prostate, and the homogeneity index ${ }^{3}$, used in reporting brachytherapy treatments, and also to judge the quality of a dose plan ${ }^{4}$.

A finding from the early clinical introduction of inverse GYN planning is that results, in terms of dosimetric indices are comparable or better when automated optimization methods are used instead of manual ${ }^{5}$. However dose plans generated by inverse planning tend to be comprised of fewer dwell positions, with correspondingly longer dwell times than manually generated plans ${ }^{5}$. The same phenomenon has been reported in the planning of prostate brachytherapy ${ }^{6}$. Concern was raised about the unknown effect of the hot spots around the corresponding positions ${ }^{5,6}$, and it was suggested that more homogeneous solutions would be preferable ${ }^{5}$. Since then different ways of reducing the long dwell times have been proposed and successfully used, e.g., by introducing artificial normal tissue around the catheters ${ }^{5}$, by introducing explicit ceilings on the maximum dwell time ${ }^{5}$, and by restricting dwell time gradients ${ }^{6}$.

One of the basic principles of inverse planning methods currently available, such as IPSA $^{1}$ and HIPO ${ }^{2}$, is the use of objective functions comprising linear penalties on undesired doses. In this article, we will show mathematically that one cause of the long dwell times, that is not observed in manually generated plans, is the linnear assignment of penalties to undesired doses. We will also suggest an alternative way of assigning penalties, that should reduce the effects of the linearity. Experiments to compare the linear and the alternative penalties are carried out retrospectively on a set of ten prostate cancer patients. It is of fundamental interest that the objective function itself is one cause for the long dwell times, even though methods such as the restriction of dwell time gradients ${ }^{6}$, can be successfully applied to mitigate them. The choice of objective function is a corner stone in any mathematical optimization model and limitations of the objectives are likely to lead to suboptimal outcomes.

The paper starts by introducing the mathematical model used in many inverse planning methods, and then continues with a mathematical analysis of this model. Readers not interested in the mathematical analysis can proceed directly to Section 2.2, where the alternative penalties are presented, or to the experimental part in Section 3, where the outcomes from the two types of penalties are compared. 


\section{Mathematical model and theory}

The anatomy based treatment planning of single stepping source HDR brachytherapy requires that important anatomical structures (irradiation target and the organs-at-risk) and catheter positions have been contoured on 3-D images. With this information the calculation of source dwell time distribution, referred to in the following as creating a dose plan, can begin.

When optimizing dose plans, the established approach is to select a number of points in each anatomical structure in the treatment volume, and calculate the doses to them. In the following these points are referred to as the dose-calculation points. The optimization model for dose planning presented below follows the work of Alterovitz et al. ${ }^{7}$. It has also been used by Lessard et al. ${ }^{1}$ and is very similar to the model used in HIPO ${ }^{2}$.

Let $S$ be the number of structures, and $N_{s}$ be the number of points representing the anatomical structure $s$. For each structure, a desired dose-range is provided, and doses outside these ranges are undesired. Denote the upper and lower limits for the dose-range for structure $s$ by $D_{s}^{\max }$ and $D_{s}^{\min }$, respectively. The desired dose at a dose-calculation point in structure $s$, should thus belong to the interval $\left[D_{s}^{\min }, D_{s}^{\max }\right]$. However, it is rarely possible to find a dose plan that gives doses within the specified range for every point. Hence, optimization models choose to penalise doses outside the specified doseranges by imposing penalties, the magnitudes of which are determined in a linear manner by the deviations from the specified ranges. In what follows, we denote the penalty per unit of violation of the lower and upper limits by $M_{s}^{\text {min }} \geq 0$ and $M_{s}^{\max } \geq 0$, respectively.

To deliver the dose, a radioactive source, usually ${ }^{192} \mathrm{Ir}$, is moved through the catheters. The source stops at certain points, called dwell positions, which are distributed along the catheters. Let $n$ be the total number of possible dwell positions. The dose-rate contribution from one dwell position, $j$, to one dose-calculation point, $i$, in structure $s$, is calculated in advance. Denote this value by $d_{i j}^{s}$. The total dose at point $i$ in structure $s$, $\operatorname{Dose}_{i}^{s}$, is thus

$$
\operatorname{Dose}_{i}^{s}=\sum_{j=1}^{n} d_{i j}^{s} t_{j},
$$

where $t_{j}$ denotes the dwell time in position $j$.

Letting $w_{i}^{s}$ denote the penalty for dose-calculation point $i$ in structure $s$ and normalizing the penalty per structure (i.e., dividing the total penalty for each structure by the number of points), the problem of minimizing the total penalty is reduced to solving the following optimization model.

Objective function

$$
\min \sum_{s=1}^{S} \sum_{i=1}^{N_{s}} \frac{w_{i}^{s}}{N_{s}}
$$

Subject to

$$
\begin{array}{ll}
w_{i}^{s}+\sum_{j=1}^{n} M_{s}^{\text {min }} d_{i j}^{s} t_{j} \geq M_{s}^{\text {min }} D_{s}^{\text {min }}, & \forall i, s \\
w_{i}^{s}-\sum_{j=1}^{n} M_{s}^{\text {max }} d_{i j}^{s} t_{j} \geq-M_{s}^{\text {max }} D_{s}^{\text {max }}, & \forall i, s
\end{array}
$$




$$
\begin{aligned}
w_{i}^{s} \geq 0, & \forall i, s \\
t_{j} \geq 0, & \forall j
\end{aligned}
$$

This optimization problem will be referred to as the linear penalty model. Since all constraints as well as the objective function are linear, it is a linear optimization problem. In the discussion below, the well-documented basic theory of linear optimization problems is frequently used. For a general reference on this subject, see for example Murty ${ }^{8}$.

\subsection{Analysis of the linear penalty model}

For linear optimization problems, it is a well-known fact that if a problem has an optimal solution, then some extreme point of the feasible set, defined here by Eqs. (2)-(5), is optimal. Extreme points have special properties, one of which is that only a certain number of variables can attain non-zero values. Below, we intend to show that this mathematical property is one cause for the long dwell times produced by the model above.

The non-zero variables are known as basic variables, and there are as many basic variables as there are linear constraints (excluding non-negativity constraints, here Eqs. (4) and (5)). When determining the number of basic variables, the problem should be in standard form, which means that it should be stated with linear equality constraints and non-negative variables. In our problem, we have inequality constraints and therefore we have to transform it into standard form in order to be able to count basic variables. This can be done by adding so-called slack variables, one for each inequality constraint. Such a variable, denoted by $s_{i s}^{\min / \max }$, measures the difference between the left-handside and the right-hand-side of an inequality constraint. Performing these changes yields the following model.

Objective function

$$
\min \sum_{s=1}^{S} \sum_{i=1}^{N_{s}} \frac{w_{i}^{s}}{N_{s}}
$$

Subject to:

$$
\begin{aligned}
w_{i}^{s}+\sum_{j=1}^{n} M_{s}^{\text {min }} d_{i j}^{s} t_{j}-s_{i s}^{\text {min }} & =M_{s}^{\text {min }} D_{s}^{\text {min }}, & & \forall i, s \\
w_{i}^{s}-\sum_{j=1}^{n} M_{s}^{\text {max }} d_{i j}^{s} t_{j}-s_{i s}^{\text {max }} & =-M_{s}^{\text {max }} D_{s}^{\text {max }}, & & \forall i, s \\
w_{i}^{s} & \geq 0, & & \forall i, s \\
t_{j} & \geq 0, & & \forall j \\
s_{i s}^{\text {min }}, s_{i s}^{\text {max }} & \geq 0, & & \forall i, s
\end{aligned}
$$

Counting constraints and variables in the model yields $2 \sum_{s=1}^{S} N_{s}$ constraints and $n+$ $3 \sum_{s=1}^{S} N_{s}$ variables. Therefore at most, $2 \sum_{s=1}^{S} N_{s}$ variables can be non-zero at an optimal extreme point. 
Consider one specific dose-calculation point, $\hat{\imath}$, in structure $\hat{s}$. At this point, two constraints apply, one according to Eq. (6) and the other according to Eq. (7). Using Eq. (1) for the point $\hat{\imath}$ in structure $\hat{s}$, the equalities associated with this point are

$$
w_{\hat{\imath}}^{\hat{s}}-s_{\hat{\imath} \hat{s}}^{\min }=M_{\hat{s}}^{\min }\left(D_{\hat{s}}^{\min }-\operatorname{Dose}_{\hat{\imath}}^{\hat{s}}\right)
$$

and

$$
w_{\hat{\imath}}^{\hat{s}}-s_{\hat{\imath} \hat{s}}^{\max }=M_{\hat{s}}^{\max }\left(\operatorname{Dose}_{\hat{\imath}}^{\hat{s}}-D_{\hat{s}}^{\max }\right) .
$$

Figure 1: Penalty function for one dose-calculation point.

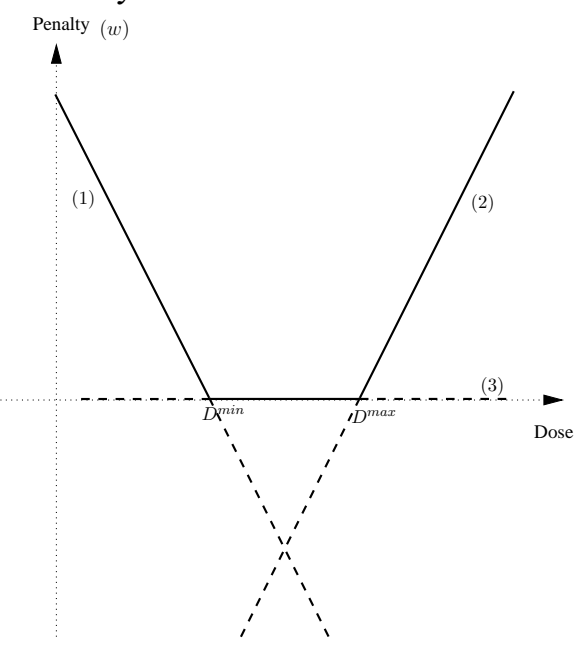

In Figure 1, these constraints correspond to segments (1) and (2) respectively. Segment (3) corresponds to the constraint that no penalty variable can be negative. Let us now consider the case when $\operatorname{Dose}_{\hat{\imath}}^{\hat{s}} \in\left[0, D_{\hat{s}}^{\text {min }}\right)$, that is, when $0 \leq \operatorname{Dose}_{\hat{\imath}}^{\hat{s}}<D_{\hat{s}}^{\text {min }}$ holds. Since $M_{\hat{s}}^{\min }>0$, the right hand side of Eq. (11) is strictly positive and hence $w_{\hat{\imath}}^{\hat{s}}$ has to be strictly positive, since all variables are positive. For Eq. (12) a similar reasoning applies: $M_{\hat{s}}^{\max }>0$ and hence the right hand side of Eq. (12) is strictly negative, forcing $s_{\hat{\imath} \hat{s}}^{\max }$ to be strictly positive. Together, these arguments imply that if $\operatorname{Dose}_{\hat{\imath}}^{\hat{s}} \in\left[0, D_{\hat{s}}^{\text {min }}\right)$, then the two variables, $w_{\hat{\imath}}^{\hat{s}}$ and $s_{\hat{\imath} \hat{s}}^{\max }$, are strictly positive and hence basic variables. The case when $\operatorname{Dose}_{\hat{\imath}}^{\hat{s}} \in\left(D_{\hat{s}}^{\max }, \infty\right)$ can be dealt with in a similar way, the conclusion then being that the two variables $w_{\hat{\imath}}^{\hat{s}}$ and $s_{\hat{\imath} \hat{s}}^{\min }$ are forced to be strictly positive and hence must be basic variables. When $\operatorname{Dos} e_{\hat{\imath}}^{\hat{s}} \in\left(D_{\hat{s}}^{\min }, D_{\hat{s}}^{\max }\right)$ the reasoning is slightly different. The righthand-sides of both Eqs. (11) and (12) are strictly negative, forcing both $s_{\hat{\imath} \hat{s}}^{\min }$ and $s_{\hat{\imath} \hat{s}}^{\max }$ to be strictly positive and hence to be basic variables. Lastly, the case when $\operatorname{Dose}_{\hat{\imath}}^{\hat{s}}=D_{\hat{s}}^{\text {min }}$ or Dose $e_{\hat{\hat{s}}}^{\hat{s}}=D_{\hat{s}}^{\max }$ (later we will refer to these cases as the dose-calculation point being at its breakpoint), then the right hand side of either Eq. (11) or Eq. (12) is zero, while the other is strictly negative. This implies that either $s_{\hat{\imath} \hat{s}}^{\min }$ or $s_{\hat{\imath} \hat{s}}^{\max }$ is strictly positive and hence one of them is a basic variable. The number of forced basic variables is summarized in Table 1, and the argument above is illustrated in Figure 2. (For one-sided 
Figure 2: Illustration of the variables that have to be basic, given the dose at point $\hat{\imath}$ in structure $\hat{s}$.

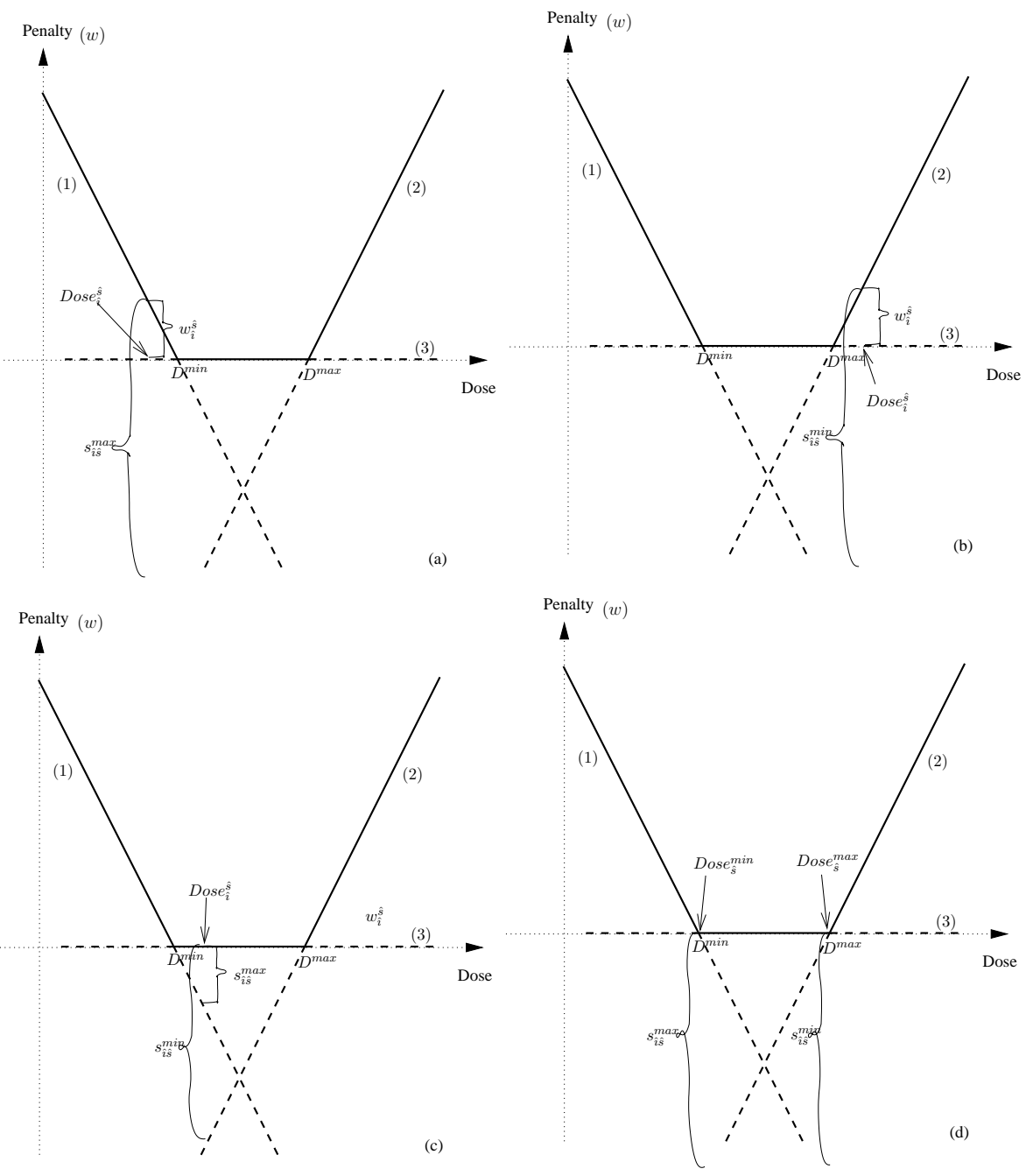

penalties, typically used for organs-at-risk, a similar analysis giving an equivalent result can be made.)

The analysis above holds for all dose-calculation points and the number of basic variables occupied by penalty variables and slack variables can therefore be easily calculated. Let $n_{d}$ be the total number of dose-calculation points with a total dose equal to the upper or the lower limit of the dose-range. Then the number of occupied basic variables is $2 \sum_{s} N_{s}-n_{d}$. This means that the maximum number of dwell time variables that can be positive is $n_{d}$. Further, for each positive dwell time, the total dose at one dose-calculation 
Table 1: Number of forced basic variables from Eq. (11) and (12).

\begin{tabular}{|ccc|}
\hline \hline Dose & Basic variables & No basic variables \\
\hline$\left[0, D_{\hat{\imath}}^{\hat{s}} \in\right.$ & $w_{\hat{\imath}}^{\hat{s}}, s_{\hat{\imath} \hat{s}}^{\max }$ & 2 \\
{$\left[D_{\hat{s}}^{\text {min }}\right]$} & $s_{\hat{\imath} \hat{s}}^{\max }$ & 1 \\
$\left(D_{\hat{s}}^{\min }, D_{\hat{s}}^{\max }\right)$ & $s_{\hat{\imath} \hat{s}}^{\min }, s_{\hat{\imath} \hat{s}}^{\max }$ & 2 \\
{$\left[D_{\hat{s}}^{\max }\right]$} & $s_{\hat{\imath} \hat{s}}^{\min }$ & 1 \\
$\left(D_{\hat{s}}^{\max }, \infty\right)$ & $w_{\hat{\imath}}^{s}, s_{\hat{\imath} \hat{s}}^{\min }$ & 2 \\
\hline
\end{tabular}

point must be equal to either the upper or the lower limit of the dose-range.

We conclude that the total number of positive dwell times is bounded by the number of dose-calculation points with a dose that coincides with their upper or lower limits. Our tests, which are the same as those for which results are presented in Table 8, show that the number of active dwell positions is exactly the same as the number of dosecalculation points that have a dose that coincides with their upper or lower limits. Hence, our observation is that in practice, relatively few doses will attain the upper or lower limits, and hence only a few dwell time variables become non-zero, so that the dose plans generated have relatively few positive dwell times.

Before continuing with our alternative penalties, we will make a remark about alternative optima ${ }^{8}$ to linear optimization problems. As is well known, linear problems may have alternative optimal solutions, in which case there are non-extreme points that are optimal. These do not have the same properties as extreme points, and in particular, more variables can be non-zero. Alternative optimal solutions that are not extreme points can be found by using specific solution techniques such as the interior point method. Our computations have shown, however, that for the patients presented in Section 3, the set of alternative solutions, when such exist, is very small. Our computations show that the maximal possible change in the value of a dwell time variable between alternative optimal solutions is about $10^{-4} s$. Therefore in practice, the set of optimal solutions seem to be so small that it is almost only one point. This implies that in order to find solutions with more homogeneous dwell times, it is not enough to choose a specific solution technique. Instead, it is necessary to change the model.

\subsection{Alternative penalty}

Since the linear penalties result in few positive dwell times, it is necessary to adjust the linear penalty model to allow more dwell positions to become active. As mentioned above, such adjustments such as the introduction of artificial normal tissue around the catheters ${ }^{5}$, explicit ceilings on maximum dwell time ${ }^{5}$, or restrictions on dwell time gradients ${ }^{6}$ have been proposed earlier. Our opinion, however, is that rather than including auxiliary constraints that limit the length of the dwell times, it is preferable to change the underlying property of the model that causes them to become too long, that is, the linearity of the penalties. Our choice for improving the dose planning model is therefore to use other types of penalties, namely convex piecewise linear penalties.

To increase the number of positive dwell times, and thereby shorten them, more dosecalculation points have to recieve doses at breakpoints. One way of achieving this is 
to introduce more breakpoints. This can be accomplished with convex piecewise linear penalty functions. A piecewise linear function consists of a number of linear segments; see Figure 3 for an example. The use of such functions introduces more breakpoints, and as long as the function is convex, the problem can be solved using the same techniques as those used for the linear penalty model. Piecewise linear penalty functions also provide an opportunity for further differentiation of the penalties, e.g., points with doses far from their required dose-ranges can be penalised more than points closer to the dose-range. Our choice is also supported by the work of Romeijn et al. ${ }^{9}$, who use piecewise linear penalties for the IMRT fluence-map optimization problem (also known as the beamletintensity optimization problem) with excellent results.

Figure 3: Convex piecewise linear function with seven segments.

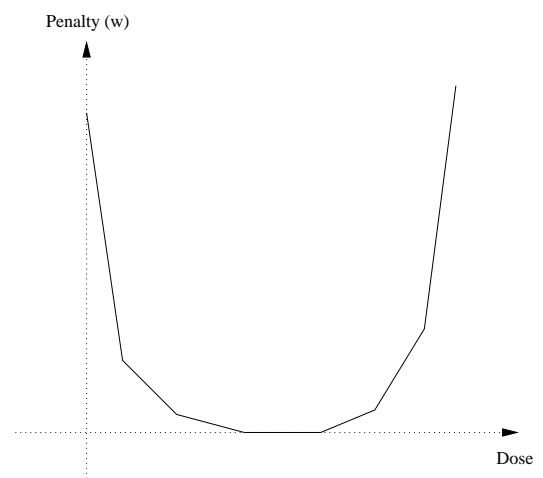

Assume that the penalty for dose-calculation points in structure $s$ is determined by a convex piecewise linear function with $k_{s}$ segments. Segment $k$ corresponds to the linear function $c_{s}^{k}+\sum_{j=1}^{n} M_{s}^{k} d_{i j}^{s} t_{j}$, where $M_{s}^{k}$ is the slope and $c_{s}^{k}$ the y-intercept. Then the alternative model becomes as follows.

Objective function

$$
\min \sum_{s=1}^{S} \sum_{i=1}^{N_{s}} \frac{w_{i}^{s}}{N_{s}}
$$

Subject to

$$
\begin{array}{ll}
w_{s}^{i} \geq c_{s}^{k}+\sum_{j=1}^{n} M_{s}^{k} d_{i j}^{s} t_{j}, & \forall i, s, k \\
w_{i}^{s} \geq 0, & \forall i, s \\
t_{j} \geq 0, & \forall j .
\end{array}
$$

The piecewise linear model can be analysed in the same way as the linear penalty model: for a dwell time to be positive the dose at one dose-calculation point has to coincide with a breakpoint. The number of breakpoints is, however, larger when using piecewise linear penalties, instead of linear, and the likelihood for a dose to coincide with a 
breakpoint increases significantly. Hence more dwell times are likely to become positive, whereby the length of the individual dwell times are likely to be reduced.

\section{Materials and methods}

For comparison of the two models, the anatomy and catheter positions from ten clinical implants for HDR brachytherapy for prostate cancer (given as a boost to external beam therapy) were selected and used retrospectively. The implants were chosen to represent a wide range of prostates with respect to both shapes and volumes. The number of catheters ranged from 16 to 20 and dwell positions were distributed in the catheters with $2.5 \mathrm{~mm}$ in-between. Some characteristics of the prostates are presented in Table 2.

Table 2: Prostate characteristics, $P D P=$ Possible dwell positions.

\begin{tabular}{|ccccc|}
\hline \hline Case No: & Volume $\left(\mathrm{cm}^{3}\right)$ & No catheters & PDP & PDPs per $\mathrm{cm}^{3}$ \\
\hline 1 & 58 & 20 & 351 & 6.1 \\
2 & 26 & 19 & 251 & 9.7 \\
3 & 25 & 19 & 220 & 8.8 \\
4 & 32 & 19 & 244 & 7.6 \\
5 & 24 & 20 & 201 & 8.4 \\
6 & 19 & 16 & 189 & 9.9 \\
7 & 35 & 20 & 265 & 7.6 \\
8 & 39 & 20 & 265 & 6.8 \\
9 & 40 & 19 & 303 & 7.6 \\
10 & 57 & 20 & 293 & 5.1 \\
\hline \hline
\end{tabular}

The target was the prostate (CTV). The urethra and rectum were considered organs-atrisk (OARs), and these were contoured on the ultrasound images by a radiation oncologist. To spare the normal tissue surrounding the prostate, an additional organ-at-risk which was neither urethra or rectum, but which instead, consisted of tissue adjacent to the prostate and at most $1 \mathrm{~cm}$ away from it, was introduced by us for this study.

For each patient, a set of dose-calculation points was chosen using the method proposed by Lahanas ${ }^{10}$. In this method, dose-calculation points should be generated both on the surface of each structure and inside the volume of each structure. A minimum number of 20 points per structure was always used however. In Table 3 , the density of the points for each structure listed is shown. The dose-rate contributions, $d_{i j}^{s}$, were calculated using functions fitted to the dose distribution in water around a single HDR ${ }^{192} \mathrm{Ir}$ source (Nucletron microSelectron HDR v1), that was initially derived by EGS4 Monte Carlo simulations ${ }^{11}$. This approach produces data on dose in water as a function of distance and polar angle with the source equivalent to that obtained using the AAPM TG43 formalism $^{12}$. For a plan to be considered satisfactory, the dosimetric protocol shown in Table 4 should be fulfilled. A similar protocol was used by Baltas et al. ${ }^{6}$. The penalty parameters for both models $\left(M_{s}^{\min }, M_{s}^{\max }, D_{s}^{\min }, D_{s}^{\max }, M_{s}^{k}\right.$, and $\left.c_{s}^{k}\right)$ were adapted to each patient, in order to obtain satisfactory treatment plans by using manual tuning methods.

To solve the two models, the state-of-the-art optimization software CPLEX ${ }^{13}$ was 
Table 3: Dose calculation point distribution.

\begin{tabular}{|clc|}
\hline \hline Structure & & Density $\left(/ \mathrm{cm}^{2}\right.$ or $\left./ \mathrm{cm}^{3}\right)$ \\
\hline \multirow{2}{*}{ Prostate } & Surface & 3 \\
& Volume & 10 \\
& Srethra & 3 \\
& Surface & 10 \\
& Volume & 3 \\
Rectum & Surface & 10 \\
& Volume & 3 \\
\hline \hline
\end{tabular}

Table 4: Dosimetric protocol for this study.

\begin{tabular}{|lc|}
\hline \hline Parameter & Value \\
\hline Reference Dose & $11.5 \mathrm{~Gy}$ \\
$D_{90}$-Prostate & $\geq 100 \%$ \\
$V_{100}$-Prostate & $\geq 90 \%$ \\
$V_{150}$-Prostate & $\leq 35 \%$ \\
$D_{10}$-Urethra & $\leq 115 \%$ \\
$D_{10}$-Rectum & $\leq 75 \%$ \\
\hline
\end{tabular}

used. The time needed to solve the linear penalty model or the alternative model depends on the number of dose-calculation points used, and for our patients, the time varied between 1 and 10 seconds (on a PC with an $3.2 \mathrm{GHz}$ Intel core i5 processor and 4GB RAM). Which of the models it was faster to solve, varied among patients, and hence the models are similar with respect to the time needed.

In comparing the plans generated by the two models, indices of the dosimetric protocol in Table 4, as well as other relevant dose-volume parameters for both the prostate and the OARs were considered.

\section{Results}

In Tables 5, 6, and 7 the results of the dosimetric analysis for prostate, urethra, rectum and surrounding normal tissue are summarized for both the linear and the piecewise linear model. Most of the dose-volume parameters are similar for the two models and they cannot be shown to be significantly better or worse when piecewise linear penalty functions are used $(p<0.05)$. However, in all cases where the dose-volume parameters are significantly different, they are better for the piecewise linear model. In the prostate (the target), the volumes receiving the highest doses are significantly reduced, as shown by an average $20 \%$ decrease in $V_{200}$ (range from 0.3 to $36.8 \%$, hence an improvement was seen for all patients) and an average $40 \%$ decrease in $V_{300}$ (range from 20 to $50 \%$ ). The dosimetric parameter $V_{150}$ was not significantly different for the two models but it did however, decrease by $6 \%$ on average, with an improvement in the case of 7 of the 10 patients. For the urethra, the dosimetric parameters $D_{10}$ and $V_{100}$ were significantly reduced and for normal surrounding tissue, $D_{1}, V_{100}$, and $V_{200}$ were significantly reduced. The low average value of $85.2 \%$ in $V_{100}$ for urethra obtained with the piecewise linear objective indicates an excessive sparing of this organ. This can be resolved by further tuning the penalty parameters to be less demanding towards urethral doses.

With regards to the impact of the models on the dwell times, the mean reduction in total dwell time was $4.2 \%$, and this was statistically significant $(\mathrm{p}=0.0004)$. Furthermore, the number of active dwell positions, that is, points with positive dwell times, increased significantly $\left(p=10^{-6}\right)$. The linear model activated on average $43.7 \%$ of the possible dwell positions, while the piecewise linear model activated on average $52.3 \%$. Thus, 
Table 5: Mean values and standard deviations for the dosimetric indices of the prostate using the linear (1) and piecewise linear (p) model.

\begin{tabular}{|ccccc|}
\hline \hline Parameter & Plan & Mean Value (\%) & Standard deviation (\%) & P-value \\
\hline \multirow{2}{*}{$D_{90}$} & $\mathrm{l}$ & 102.5 & 2.6 & 0.540 \\
& $\mathrm{p}$ & 102.1 & 1.7 & \\
$D_{95}$ & 1 & 95.4 & 3.2 & 0.760 \\
& $\mathrm{p}$ & 95.7 & 2.1 & \\
$D_{100}$ & $\mathrm{l}$ & 73.8 & 3.4 & 0.183 \\
& $\mathrm{p}$ & 72.0 & 5.0 & \\
$V_{100}$ & 1 & 92.0 & 1.9 & 0.908 \\
& $\mathrm{p}$ & 91.9 & 1.5 & \\
$V_{150}$ & 1 & 15.4 & 3.0 & 0.206 \\
& $\mathrm{p}$ & 14.4 & 2.8 & 0.001 \\
$V_{200}$ & 1 & 5.2 & 1.1 & 0 \\
& $\mathrm{p}$ & 4.1 & 0.9 & \\
$V_{300}$ & 1 & 1.6 & 0.4 & \\
& $\mathrm{p}$ & 1.0 & 0.3 & \\
\hline
\end{tabular}

Table 6: Mean values and standard deviations for the dosimetric indices of the urethra using the linear (l) and piecewise linear ( $p$ ) model.

\begin{tabular}{|ccccc|}
\hline \hline Parameter & Plan & Mean Value (\%) & Standard deviation (\%) & P-value \\
\hline \multirow{2}{*}{$D_{1}$} & $\mathrm{l}$ & 115.5 & 3.3 & 0.097 \\
& $\mathrm{p}$ & 113.7 & 2.8 & \\
$D_{10}$ & 1 & 111.7 & 3.3 & 0.033 \\
& $\mathrm{p}$ & 110.2 & 2.8 & \\
$V_{100}$ & 1 & 98.6 & 1.9 & 0 \\
& $\mathrm{p}$ & 85.2 & 4.5 & 0.071 \\
$V_{115}$ & 1 & 3.2 & 4.0 & \\
& $\mathrm{p}$ & 0.8 & 1.4 & \\
\hline \hline
\end{tabular}

around $20 \%$ more dwell positions were active with the piecewise linear model. One of the major differences with the piecewise linear model is a reduction of the maximum dwell time, by on average $25 \%$ (significant at level $\mathrm{p}=0.015$ ). In Table 8 , total dwell time, maximum dwell time, and the number of active dwell positions are listed for the ten patients. It can be seen that both total dwell time and the number of activated dwell positions are improved for all patients, and the maximum dwell time is improved for all but one patient. Figure 4 presents a representative comparison of dwell time distributions for the two models, and has been obtained from patient number 7 . 
Table 7: Mean values and standard deviations for the dosimetric indices of the rectum and surrounding normal tissue using the linear (l) and piecewise linear ( $p$ ) model.

\begin{tabular}{|ccccc|}
\hline \hline Parameter & Plan & Mean Value (\%) & Standard deviation (\%) & P-value \\
\hline \multirow{2}{*}{$D_{10}^{\text {rectum }}$} & $\mathrm{l}$ & 49.5 & 3.7 & 0.495 \\
& $\mathrm{p}$ & 49.8 & 3.3 & \\
$D_{10}^{\text {normal }}$ & 1 & 86.5 & 16.0 & 0 \\
& $\mathrm{p}$ & 83.5 & 15.6 & \\
$V_{100}^{\text {normal }}$ & $\mathrm{l}$ & 7.1 & 4.6 & 0.005 \\
& $\mathrm{p}$ & 6.4 & 4.3 & 0 \\
$V_{200}^{\text {normal }}$ & $\mathrm{p}$ & 0.5 & 0.3 & 0 \\
\hline \hline
\end{tabular}

Table 8: Comparision of total dwell time, maximum dwell time and number of activated dwell positions for the ten patients using the linear (l) and piecewise linear (p) model.

\begin{tabular}{|ccccccc|}
\hline \hline $\begin{array}{c}\text { Case } \\
\text { No: }\end{array}$ & \multicolumn{2}{c}{$\begin{array}{c}\text { Total dwell } \\
\text { time }(\mathrm{s})\end{array}$} & \multicolumn{2}{c}{$\begin{array}{c}\text { Maximum dwell } \\
\text { time }(\mathrm{s})\end{array}$} & \multicolumn{2}{c|}{$\begin{array}{c}\text { Active dwell } \\
\text { positions }(\%)\end{array}$} \\
& 1 & $\mathrm{p}$ & 1 & $\mathrm{p}$ & $\mathrm{l}$ & $\mathrm{p}$ \\
\hline 1 & 680 & 666 & 33.4 & 23.5 & 47.8 & 55.8 \\
2 & 452 & 436 & 42.9 & 28.7 & 41.4 & 53.8 \\
3 & 480 & 458 & 57.8 & 28.6 & 41.8 & 52.7 \\
4 & 511 & 495 & 54.9 & 30.2 & 44.3 & 51.6 \\
5 & 488 & 480 & 41.2 & 43.9 & 40.3 & 50.8 \\
6 & 441 & 425 & 50.5 & 46.0 & 40.2 & 48.2 \\
7 & 543 & 505 & 60.3 & 21.7 & 46.0 & 57.7 \\
8 & 570 & 533 & 29.9 & 29.7 & 45.3 & 52.5 \\
9 & 561 & 543 & 34.1 & 30.7 & 45.9 & 51.5 \\
10 & 767 & 715 & 50.7 & 43.2 & 43.7 & 48.5 \\
\hline \hline
\end{tabular}

As mentioned above, the results presented here are based on one dwell position per 2.5 $\mathrm{mm}$ in the catheter. Calculations have also been performed with both models using a stepdistance of $5 \mathrm{~mm}$. With this larger step, the results were not as easily interpreted as those presented above, and fewer significant differences between the models were observed. The reason for this is that with a $5 \mathrm{~mm}$ step-size, the activation of the dwell positions is already very high for the linear model (70\% of the dwell positions were activated), since almost all of them are needed to reach an acceptable overall dose. The piecewise linear model is therefore not able to activate many more dwell positions, and hence the results of the two models become very similar. 
Figure 4: Dwell time distributions for patient number 7 for the two different models.

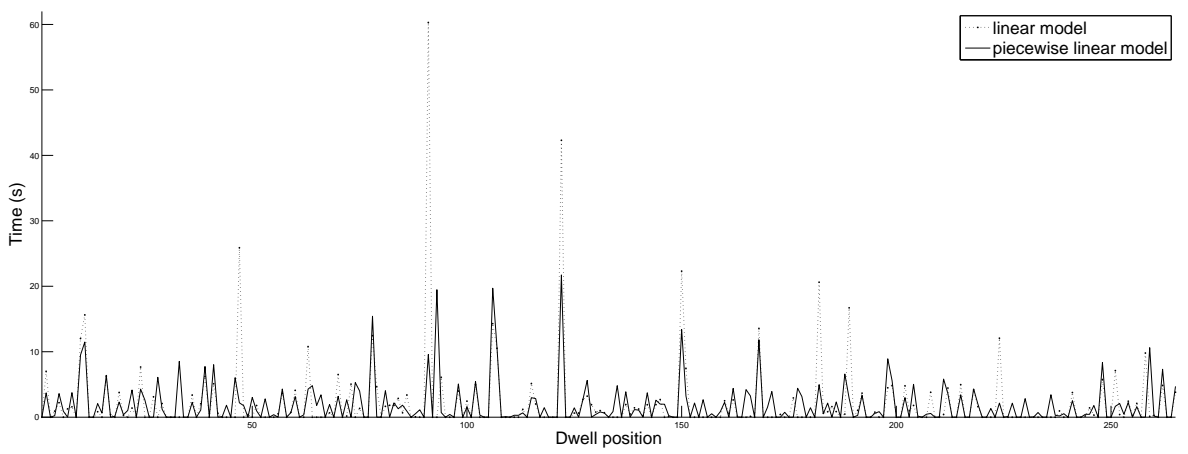

\section{Summary and conclusion}

One of the basic principles of optimization techniques for anatomy based inverse planning of HDR brachytherapy, is the use of objective functions comprising linear penalties on undesired doses ${ }^{1,2}$. Dose plans generated with these techniques have shown a tendency to be comprised of fewer and longer dwell times than manually generated plans. Concern has been raised against the hot spots caused by long dwell times ${ }^{5}$ which has led to the development of various methods to mitigate them, such as the restriction of dwell time gradients ${ }^{6}$ or the use of explicit ceilings on dwell times ${ }^{5}$. In this paper, we have shown that one cause for the long dwell times is precisely the use of objectives comprising linear penalties on undesired doses. Optimal solutions to the linear model correspond to extreme points which have a very restricted number of positive variables. This leads to the conclusion that only as many dwell times as there are dose-calculation points on their upper or lower dose-limits can be positive. Since it is not likely that a dose-calculation point is on a breakpoint there will be relatively few positive dwell times, and in order to reach the desired overall dose level, some of these will become long.

Therefore adjustments of the optimization model are needed to allow more dwell positions to become active, and indirectly, this allows the dwell times to become shorter. In order to achieve this we introduced piecewise linear penalties that reduce the long dwell times by introducing more breakpoints. By introducing more breakpoints, the likelihood of a dose coinciding with a breakpoint increases, and hence more dwell times are likely to become positive, thereby reducing the length of the individual dwell times. Furthermore piecewise linear penalties make it possible to differentiate the penalty more than simple linear penalties are able to do.

Experimental comparisons between the two types of penalties were carried out retrospectively on a set of prostate cancer patients. The comparisons show a significant change in dwell times when the piecewise linear model is used, with a $4 \%$ average total time reduction, and a $20 \%$ average increase in the number of dwell positions activated. The maximum dwell time is reduced by an average of $25 \%$. Dosimetric indices were very sim- 
ilar for the two models, except for slight reductions in high doses to the prostate, urethra, and surrounding normal tissue. Hence a change to the piecewise linear penalty suggested here effectively mitigates the longest source dwelling times, but yields only moderate improvements in clinical DVH parameters. Although it has been shown that methods other than a change of objective can also be used to reduce source dwelling times, this work show the interesting result that the fewer but longer dwell times observed when using mathematical optimization methods instead of manual planning in brachytherapy stems from the simplicity of the objective function used.

\section{References}

[1] E. Lessard, and J. Pouliot, "Inverse planning anatomy-based dose optimization for HDR-brachytherapy of the prostate using fast simulated annealing algorithm and dedicated objective function," Med. Phys. 28, 773-779 (2001).

[2] D. Baltas, E. Colla, A. Karabis, and C. Kirisits, "Optimization and inverse planning tools in oncentra gyn," Available: http://www.nucletron.com/en/ ProductsAndSolutions/Pages/OncentraGYN . aspx (downloaded october 2010).

[3] G. C. Morton, R. Sankreacha, P. Halina and A. Loblaw, "A comparison of anatomybased inverse planning with simulated annealing and graphical optimization for highdose-rate prostate brachytherapy," Brachytherapy 7, 12-16 (2008).

[4] G. Kovács, R. Pötter, T. Loch, J. Hammer, I.-K. Kolkman-Deurloo, J. J. de la Rosette, and H. Bertermann, "GEC/ESTRO-EAU recommendations on temporary brachytherapy using stepping sources for localised prostate cancer," Radiotherapy and Oncology 74(2), 137-148 (2005).

[5] E. Chajon, I. Dumas, M. Touleimat, N. Magné, J. Coulot, R. Verstraet, D. Lefkopoulos, and C. Haie-Meder, "Inverse planning approach for 3-D MRI-based pulse-dose rate intracavitary brachytherapy in cervix cancer," Int. J. Radiat. Oncol., Biol., Phys. 69, 955-961 (2007).

[6] D. Baltas, Z. Katsilieri, V. Kefala, S. Papaioannou, A. Karabis, P. Mavroidis, and N. Zamboglou, "Influence of modulation restriction in inverse optimization with hipo of prostate implants on plan quality: Analysis using dosimetric and radiobiological indices," IFMBE Proceedings 25, 283-286 (2009).

[7] R. Alterovitz, E. Lessard, J. Pouliot, I. C. Hsu, J. F. O’Brien, and K. Goldberg, “Optimization of HDR brachytherapy dose distributions using linear programming with penalty costs," Med. Phys. 33, 4012-4019 (2006).

[8] K. G. Murty, “Linear programming,” Wiley, New York (1983).

[9] H. E. Romeijn, R. K. Ahuja, J. F. Dempsey, A. Kumar, and J. G. Li, "A novel linear programming approach to fluence map optimization for intensity modulated radiation therapy treatment planning," Phys. Med. Biol. 48, 3521-3542 (2003). 
[10] M. Lahanas, D. Baltas, S. Giannouli, N. Milickovic, and N. Zamboglou, "Generation of uniformly distributed dose points for anatomy-based three-dimensional dose optimization methods in brachytherapy," Med. Phys. 27, 1034-1046 (2000).

[11] K. R. Russell, Å. K. Carlsson Tedgren, and A. Ahnesjö, "Brachytherapy source characterization for improved dose calculations using primary and scatter dose separation," Med. Phys. 32, 2739-2752 (2005).

[12] M. J. Rivard, B. M. Coursey, L. A. DeWerd, W. F. Hanson, M. S. Huq, G. S. Ibbott, M. G. Mitch, R. Nath, and J. F. Williamson, "Update of AAPM Task Group No. 43 Report: A revised AAPM protocol for brachytherapy dose calculations," Med. Phys. 31, 633-674 (2004).

[13] ILOG CPLEX V12.2 User's manual for CPLEX (2010). 